\title{
Psychiatric aspects of AIDS: Overview for the general practitioner
}

JOYCE M. JOHNSON, D.O., FAOCPM

Chevy Chase, Maryland

As the acquired immunodeficiency syndrome (AIDS) epidemic spreads, many general practitioners are becoming involved in the care of these patients. Because of their complex mix of medical, psychiatric, and other problems, patients require the holistic approach offered by osteopathic medicine. AIDS patients are at special risk for psychiatric illness because of the complicated pathophysiology, sudden onset, extremely poor prognosis, and social implications of the disease. Many psychiatric conditions (organic brain syndromes, adjustment disorders, depression) are caused by AIDS, while others are closely associated with it (history of intravenous drug use). Many AIDS patients have pre-existing psychiatric illnesses (schizophrenia, affective and personality disorders), which are exacerbated by the psychosocial stress and physical limitations imposed by AIDS. This paper provides an overview of psychiatric and relevant medical issues for general practitioners and other osteopathic physicians who treat AIDS patients.

General practitioners increasingly are caring for the medical needs of patients with acquired immunodeficiency syndrome (AIDS), and they are becoming more knowledgeable about the physical aspects of the disease and its consequences. However, the high prevalence of frank psychiatric illness among AIDS patients also requires physicians to be familiar with the emotional consequences of the disease upon the patient, his/her family and friends, and the community, so that adequate treatment (and prevention) can be provided. These problems may be overlooked as the health team focuses on one medical emergency after another, and the patient may be left to manage resulting emotional traumas and conflicts alone.
The osteopathic physician should consider the patient's emotional as well as medical needs when applying osteopathic principles to the development of the patient's total treatment plan. This paper provides an overview of the epidemiology and clinical aspects of the AIDS syndrome as related to the psychiatric illnesses associated with it.

\section{Epidemiology}

Between the spring of 1981, when the first case of an illness now meeting the Centers for Disease Control (CDC) surveillance definition of AIDS $^{1}$ was reported, and November 24, 1986, a total of 27,767 adult and 402 pediatric cases of AIDS were reported to CDC from within the United States. ${ }^{2}$ Ninetythree percent of adult patients were male and 7 percent were female; nearly one half were between 30 and 39 years of age. Adult patients belonged to one of several groups: homosexual and bisexual males (65 percent); users of intravenous drugs (17 percent); homosexual males and intravenous drug users ( 8 percent); heterosexual cases ( 4 percent); recipients of transfusions and blood components ( 2 percent); and patients with hemophilia or other coagulation disorders ( 1 percent). Of the pediatric patients (children under 13 years of age at time of diagnosis), 79 percent had one or more parents with AIDS or at increased risk for the disease, 14 percent had received blood transfusions, 5 percent were patients with hemophilia or other coagulation disorders. Three percent belonged to none of these groups.

AIDS patients are at special risk for psychiatric illness because of the often sudden onset, poor prognosis, complex pathophysiology, and social implications of the disease. One retrospective review ${ }^{3}$ of 52 AIDS patients found that the chart of each made reference to neuropsychiatric disorders; 83 percent experienced a mood disturbance during their acute hospitalization, 65 percent showed signs of an organic mental syndrome, and approximately 13 percent had pre-existing psychiatric illnesses. Psychiatric consultation had been requested for $10 \mathrm{pa}-$ tients (19 percent). Only one received a psychiatric diagnosis (chronic undifferentiated schizophrenia) upon discharge. This study was important because 
it began to identify the emotional and psychiatric needs of AIDS patients.

\section{Clinical aspects}

Patients' psychiatric illnesses reflect the clinical characteristics of AIDS, which is not a discrete disease, but which by definition ${ }^{1,4}$ is a syndrome that includes any one of a number of selected opportunistic infections and tumors in a person with no known underlying cause for cellular immune deficiency. As shown in Table 1, the range of presenting infections (parasitic, fungal, bacterial, and viral) is broad. The signs of these infections include organic brain syndromes and other psychiatric illnesses (as defined by the American Psychiatric Association ${ }^{5}$ ).

Recent research ${ }^{6,7}$ has shown that the underlying immune deficiency is caused by a retrovirus, human immunodeficiency virus (HIV). In March 1985, the U.S. Food and Drug Administration ap-

TABLE 1. OPPORTUNISTIC INFECTIONS AND TUMORS MEETING THE AIDS CASE DEFINITION (FROM CDC ${ }^{1,4}$ ).

Protozoal and helminthic infections

(1) Cryptosporidiosis (causing diarrhea for longer than 1 month)

(2) Pneumocystis carinii pneumonia

(3) Toxoplasmosis (pneumonia or central nervous system disease)

Fungal infections

(1) Aspergillosis (central nervous system or disseminated disease)

(2) Candidiasis (esophagitis)

(3) Cryptococcosis (pulmonary, central nervous system, or disseminated infection

Bacterial infections

(1) Atypical mycobacteriosis (species other than tuberculosis or lepra causing disseminated infection)

Viral infections

(1) Cytomegalovirus (pulmonary, gastrointestinal tract, or central nervous system disease)

(2) Herpes simplex virus (mucocutaneous infections with ulcers lasting longer than 1 month, or pulmonary, gastrointestinal, or disseminated infection)

Cancer

(1) Kaposi's sarcoma (in person younger than 60 years of age)

(2) Lymphoma limited to the brain

If the patient has a positive serologic or virologic test for HIV but none of the above infections or tumors, the following diseases may also be reported to be AIDS:

(1) Disseminated histoplasmosis

(2) Isosporiasis (causing chronic diarrhea for longer than 1 month)

(3) Bronchial or pulmonary candidiasis

(4) Non-Hodgkin's lymphoma of high-grade pathologic type (diffuse, undifferentiated) and of B-cell or unknown immunologic phenotype

(5) Kaposi's sarcoma in patients who are 60 years of age or older at diagnosis proved an ELISA serologic test to identify antibodies to HIV, the test is now available nationwide. ${ }^{8}$ The test was developed primarily to screen donated blood for viral exposure. However, it is also being used to identify other persons who may have been exposed to the virus, particularly those at high risk. Although it is specific for antibodies to HIV and identifies those exposed to the virus, neither this test nor any other can identify the up to 30 percent of infected persons who will develop overt AIDS. ${ }^{9}$ Further, because the test detects antibodies, not the virus, it does not measure the patient's infectivity or ability to spread the disease to others. However, for preventive purposes, persons who are antibody-positive should be considered as potentially infectious. In one study ${ }^{10}$ that compared antibody status with viral culture isolates, none $(0 / 70)$ of the antibody-negative patients had positive viral culture results, while 60 percent $(43 / 72)$ of those with detectable antibodies were culture positive as well. The ability to detect exposure to HIV without a concurrent ability to predict who will develop AIDS or to identify those who are infectious, combined with the other uncertainties associated with the disease, results in dramatic emotional effects on AIDS patients and others at risk for the disease.

The clinical course of AIDS is variable, although in retrospect the patient often can describe prodromal symptoms, including weight loss, lethargy, anorexia, diarrhea, generalized lymphadenopathy, intermittent fevers, and various vague symptoms. Because most of these symptoms are nonspecific and common in non-AIDS illnesses, they are frequently experienced by persons at risk for AIDS (and others) who do not have the disease. Many minor illnesses that mistakenly are thought to be secondary to AIDS become psychosocial stressors for anxiety, depression, hypochondriasis, or suicidal ideations.

In persons developing overt AIDS, the prodrome is followed by signs and symptoms specific to HIV infection and to the patient's particular opportunistic infection or tumor, as in the following examples: Pneumocystis carinii (pneumonia); herpes simplex virus (invasive, excruciatingly painful anal or genital lesions); cryptosporidiosis (chronic treatment-resistant diarrhea); Kaposi's sarcoma (darkened, painless skin lesions); and Toxoplasma gondii (dementia or delirium). The clinical presentation of AIDS is dependent upon these secondary infections and tumors. Many can be treated, but because the underlying immune deficiency remains, the patient usually develops recurrent in fections, which are increasingly difficult to treat, and requires repeated hospitalizations. Prognosis 
is extremely grave; the disease is usually terminal within 2 years. The AIDS patient requires the same emotional support and psychiatric care common to patients with other terminal diseases that affect previously healthy young adults.

The true clinical course of infection with HIV is variable, with many infections being asymptomatic or mildly symptomatic (limited to the prodrome), while up to 30 percent will develop into syndromes meeting the case definition for AIDS. This range of clinical presentations, the inability to predict the outcome of infection in a given patient, and the seriousness of overt AIDS adds to the fear, anxiety, and guilt associated with the disease. Many homosexual males wonder if the men they love may be transmitting disease to them, or if they may be asymptomatic carriers spreading the disease to others. These concerns can result in psychosexual and other disorders meeting DSM-III criteria.

The incubation period for AIDS, or the time from exposure to clinical disease, varies from several months to several years. Even when a person ceases homosexual activity, drug use, or other behavior that placed him/her at risk for AIDS, he/she cannot be certain for years whether he/she is really well or harboring infection and may become ill in the near future. These fears and uncertainties can become psychosocial stressors to underlying personality disorders, affective disorders, and substance abuse patterns.

The characteristics of the clinical course of AIDS, then, contribute to the patterns of psychiatric illnesses related to it.

\section{Psychiatric illnesses \\ associated with AIDS}

The psychiatric illnesses of AIDS patients fall within two general classifications: (1) those disorders that pre-existed the onset of AIDS and that are not causally related to it; and (2) those disorders that are secondary to AIDS or in which AIDS is a contributory psychosocial stressor.

Pre-existing psychiatric illnesses include both those related and unrelated to substance use. Users of illicit injectable drugs comprise about 25 percent of the reported AIDS patients, including those hierarchically reported only as intravenous drug users and those homosexual and bisexual men who admitted to intravenous drug use prior to illness. ${ }^{11}$ Conversely, serologic prevalence studies show that in some geographic areas nearly 90 percent of intravenous drug users have positive antibodies to $\mathrm{HIV}^{12}$ A study of specific drug use patterns and DSM-III diagnoses of this population of AIDS patients has not been undertaken. ${ }^{13}$ However, it can be assumed that many of these patients would meet the DSM-III diagnostic criteria for one or more substance use disorders, including abuse, dependence, withdrawal, or intoxication.

Some AIDS patients have pre-existing psychiatric syndromes that are not directly associated with AIDS, such as schizophrenia, some affective disorders, and personality disorders. Although the etiology of these illnesses may be unrelated to AIDS, the concurrent diagnosis of AIDS has implications on the natural history of these mental illnesses and their treatment in a given patient. Learning that one has AIDS, with the subsequent self-identity and social changes it demands, can provide the stress that complicates the outcome of any pre-existing mental illness. Further, the physical deterioration that accompanies AIDS and the toxicity related to the pharmacologic treatment of associated infections and tumors can demand changes of the medications used to treat the patient's psychiatric condition.

Recent research ${ }^{14-16}$ shows that HIV infects the nervous system and may directly cause neuropsychiatric illness. The virus has been isolated from the cerebrospinal fluid, the spinal cord, and peripheral nerves. There is much evidence to suggest that this neurotropic virus infects brain tissue without neurologic deficits in some cases; in others it may be responsible for progressive neurologic disease. It seems to be directly related to acute and chronic meningitis, myelopathy, peripheral neuropathy, spinal cord degeneration, and chronic progressive dementia.

The opportunistic infections and tumors related to AIDS can also result in organic mental disorders that meet DSM-III criteria. ${ }^{17-20}$ Some are apparent at the time of the initial AIDS diagnosis, while others develop later in the disease process. Delirium and dementia can be caused by nearly all of the AIDS-related infections and tumors, although the causal mechanisms differ. Examples of the mechanisms include anoxia (Pneumocystis carinii pneumonia), metabolic imbalances (cryptosporidial diarrhea), encephalitis (cytomegalovirus), meningitis (cryptococcus), and direct central nervous system diseases (aspergillosis and toxoplasmosis). AIDS-related space-occupying lesions include both brain abscesses (caused by toxoplasmosis, Candida albicans, and other infections) and tumors (primary lymphoma of the brain). As with any spaceoccupying lesion, the clinical presentation varies with the size and anatomic site of the lesion. For example, frontal lobe lesions have resulted in personality changes. Organic mental disorders marked by hallucinations and delusions have been caused by several different infections.

In some patient groups, particularly the Haitian 
entrants, diagnosis of organic hallucinosis and organic delusional syndromes must be made within a cultural context. Many Haitian patients have religious beliefs that daily events are controlled by magical rituals and that illness and death are caused by spells and hexes. Spirits are generally considered an important life force. When Haitian patients relate these beliefs to physicians in the United States, they may at first seem delusional. However, if interpreted within their cultural and social frameworks, where science is not the basis of causality, the mental status of these patients can be assessed more accurately.

Illnesses of psychogenic origin are frequent complications of AIDS, because the patient undergoes many tragic experiences and is forced to cope with innumerable psychosocial stressors. ${ }^{21}$ Many homosexual patients have not completed "coming out," and learning that they have AIDS often catalyzes this process prematurely. Feelings of guilt related to one's lifestyle or the possible transmission of disease to another person may predominate. Rage and anger may be directed toward one's sexual partner(s), as a possible source of one's own disease. The transmissibility of the disease has implications for the patient's own future sexual and drug use behaviors, and he/she may not want to accept the recommended precautions. Sexual dysfunction may occur as an expression of these unresolved conflicts. Overwhelming despair, which may lead to suicide, can result as the patient is forced to deal with these social issues, the poor prognosis of the disease, and the related issues of death and dying. Further, the patient may be almost abandoned by family and friends, who fear that the disease may be transmitted to them. Unjust social discrimination only compounds the other problems these patients have.

\section{Comment}

The osteopathic physician with his/her holistic approach to care has much to offer the AIDS patient. As the number of cases increases, and as AIDS becomes prevalent in more geographic areas of the country, osteopathic physicians will increasingly be called upon to treat these patients. Therefore, all general practitioners should become knowledgeable about the medical and psychiatric aspects of the disease. Psychiatric illnesses in AIDS patients are varied, and include those caused by AIDS, those associated with it, and those that may have predated the diagnosis. Those professionals providing the patient's psychiatric and medical care should be aware of the extreme psychosocial stresses resulting from AIDS and sensitive to the specific needs of the patient. Utilizing osteopathic principles, they should work together to provide the patient with continuous and comprehensive care.

1. Centers for Disease Control: Case definition of AIDS used by CDC for epidemiologic surveillance. CDC, Atlanta, March 15, 1983

2. AIDS Activity, Centers for Disease Control: Acquired immunodeficiency syndrome weekly surveillance report-U.S. CDC, Atlanta, November 24, 1986

3. Perry, S., and Tross, S.: Psychiatric problems of AIDS inpatients at the New York Hospital. Preliminary report. Public Health Rep 99:200-5, Mar-Apr 84

4. Centers for Disease Control: Revision of the case definition of acquired immunodeficiency syndrome for national reporting-United States. MMWR 34:373-5, 28 Jun 85

5. American Psychiatric Association: Diagnostic and statistical manual of mental disorders (DSM-III). Ed. 3. APA, Washington, D.C., 1980

6. Barre-Sinoussi, F, et al.: Isolation of a T-lymphotropic retrovirus from a patient at risk for acquired immune deficiency syndrome (AIDS). Science 220:868-71, May 83

7. Gallo, R.C., et al.: Frequent detection and isolation of cytopathic retroviruses (HTLV-III) from patients with AIDS and at risk for AIDS. Science 224:500-3, May 84

8. Centers for Disease Control: Results of T-lymphotropic virus type III test kits reported from blood collection centers-United States, April 22 May 19, 1985. MMWR 34:375-6, 1985

9. Jaffe, H.W., et al.: Outcome of HTLV-III/LAV infection in a cohort of gay men. Presented at NIH Consensus Development Conference, Wash ington, D.C., July 7, 1986

10. Centers for Disease Control: Update. Public Health Service workshop on human T-lymphotropic virus type III antibody testing-United States. MMWR 34:477-8, 1985

11. Centers for Disease Control: Surveillance data. CDC, Atlanta, 1986 12. DesJarlais, D., et al.: The epidemiology of AIDS. New York State Division of Substance Abuse Services study. As reported by Centers for Disease Control. CDC, Atlanta, April 15, 1985

13. Ginzburg, $\mathrm{H}$.: Intravenous drug users and the acquired immune deficiency syndrome. Public Health Rep 99:206-12, Mar-Apr 84

14. Black, P., et al.: HTLV-III, AIDS, and the brain (editorial). N Engl J Med 313:1538-40, 12 Dec 85

15. Resnick, L., et al.: Intra-blood-brain-barrier synthesis of HTLV-IIIspecific IgG in patients with neurologic symptoms associated with AIDS or AIDS-related complex. N Engl J Med 313:1498-504, 12 Dec 85

16. Ho, D., et al.: Isolation of HTLV-III from cerebrospinal fluid and neural tissues of patients with neurologic syndromes related to the acquired immunodeficiency syndrome. N Engl J Med 313:1493-7, 12 Dec 85 17. Snider, W.D., et al.: Neurological complications of acquired immune deficiency syndrome. Analysis of 50 patients. Ann Neurol 14:403-18, Oct 83

18. Hoffman, R.S.: Neuropsychiatric complications of AIDS. Psychosomatics 25:393-400, May 84

19. Loewenstein, R.J., and Sharfstein, S.S.: Neuropsychiatric aspects of acquired immune deficiency syndrome. Int J Psychiatry Med 13:255-60, 1983-84

20. Forstein, M.: The psychosocial impact of the acquired immunodeficiency syndrome. Semin Oncol 11:77-82, Mar 84

21. Furstenberg, A.L., and Olson, M.M.: Social work and AIDS. Social Work Health Care 9:45-62, Summer 84

Accepted for publication in June 1986. Updating, as necessary, has been done by the author.

Dr. Johnson is board certified in preventive medicine. She has performed extensive research on the epidemiologic aspects of AIDS and is currently involved in the psychiatric care of patients with AIDS.

Dr. Johnson, 5518 Western Avenue, Chevy Chase, Maryland 20815. 